\title{
Cellular ceramic architectures produced by hybrid additive manufacturing: a review on the evolution of their design
}

\author{
Marco PELANCONI ${ }^{1,2}$, Ehsan REZAEI ${ }^{1}$ and Alberto ORTONA ${ }^{1, \dagger}$ \\ ${ }^{1}$ University of Applied Sciences (SUPSI): Mechanical Engineering and Materials Technology Institute (MEMTi), \\ Via Cantonale 2c, 6928 Manno, Switzerland \\ ${ }^{2}$ Department of Industrial Engineering, University of Padova, via Marzolo 9, Padova 35131, Italy
}

\begin{abstract}
We present in this work a review on the developments of computer aided design (CAD) for ceramic cellular architectures. From the first attempts in using CAD to explain by finite element modelling the behaviour of ceramic foams, this practice became a fundamental step with the newcomer ceramic additive manufacturing (AM) technique. Suddenly AM allowed the realization of such complex structures. End users requirements in several industrial fields could be satisfied thanks to the combination of CAD, simulation and AM. As structures became more complex, new tools were developed in order to solve multi-physical tasks. We first developed a tool to generate and crop periodic and random architectures. Digitally connecting the boundary struts allowed then to increase their mechanical strength. Further tools were developed to obtain periodic and random architectures with variable porosity and pore sizes. We also present an innovative tool, inspired from the atoms patterns within the grain boundaries, which allows to create a solution of continuity between two different periodic architectures. The final chapter presents a set of real components which were produced and tested in different industrial fields. (C2020 The Ceramic Society of Japan. All rights reserved.
\end{abstract}

Key-words : Additive manufacturing, Cellular ceramics, Design guideline, Lattice design, Voronoi

[Received March 27, 2020; Accepted May 7, 2020]

\section{Introduction}

Open-cell cellular structures can be idealized as a network of edges (i.e. struts) belonging to interconnected polyhedra filling the space. In nature, they exist in different forms. ${ }^{1)}$ Nowadays porous materials (polymeric, metallic and ceramic) are industrially produced and employed in different technological fields. ${ }^{2}$ ) The exploitation of these structures is a result of their outstanding properties at elevated temperatures, their stability in harsh environments (acidic, alkaline or oxidative), and superior thermomechanical properties (thermal shock resistance). Additionally, due to their porous nature, they exhibit high surface area and a percolating fluid phase, making them particularly interesting for applications such as catalysis, concentrated solar energy, thermal storage, heat exchange, radiant burners and reforming. ${ }^{3)-7)}$ Ceramic foams were first manufactured by fugitive pore forming, direct foaming, and replica of polymeric foams. ${ }^{8), 9)}$

In the recent years a new technique, named additive manufacturing (AM), has been more and more adopted to produce net shape objects. AM is a term (defined by the ASTM F2792 standard) which refers to the process of

Corresponding author: A. Ortona; E-mail: alberto.ortona@ supsi.ch

\$ Preface for this article: Dol http://doi.org/10.2109/jcersj2. 128.P9-1 joining materials' layers to make objects from a THREEDIMENSIONAL (3D) Computer-Aided Design (CAD) file. It is the opposite of the term "subtractive manufacturing" in which the material is removed by a tool. Synonyms are rapid prototyping, additive fabrication, 3D printing, additive techniques, layer manufacturing, and freeform fabrication. AM allows to produce metal, plastic and ceramic objects, we will focus our review on AM of ceramics (oxides and carbides) with complex architectures. The recent developments in AM of ceramics enable the production of structures with different morphologies. ${ }^{10)-12)}$ 3D printing techniques allows fabricating complex shapes with micro-scale details. They are vat polymerization, powder bed fusion and binder jetting. ${ }^{13)-15)}$

AM has several limitations, there is not a technique that can process every material in every shape. One way to overcome these limitations is to use hybrid AM.

Hybrid AM is the combination of new AM techniques with standard manufacturing processes of ceramic. As an example, in Ortona et al. [10] 3D printed plastic templates which were coated by replica with SiC slurries and finally infiltrated with molten Silicon.

The CAD of porous structures was first conceived to numerically explain the behaviour of foams. ${ }^{16)-18)}$ In a second step CAD was used optimize the effective properties of 3D printed porous architectures. ${ }^{19), 20)}$ In the last years these structures have been evolving incorporating 
more functionalities. Inevitably such structures demand new design tools.

A simplified model of foams was first introduced by Ashby and Gibson. ${ }^{2)}$ They assumed each cell to be "cube like". Their idea of using cubic lattice structures was introduced to explain the behaviour of foams, with analytical models, in terms of pressure drop, heat and mass transfer, and stiffness. ${ }^{21)-23)}$ Open-cell foams were later described by more complex unit-cells such as tetrakaidecahedron and Weaire-Phelan's. ${ }^{24)}$

Compared to natural foams, lattice structures are regular and reproducible. Furthermore they offer more design freedom, which results in structures with enhanced properties and novel functionalities. ${ }^{25)}$ While in the ' 90 s there was a large body of literature on the behaviour of ceramic foams, there were few studies on lattices. Deshpande et al. gave an important contribution to analytical study on their mechanical properties. ${ }^{26)}$ They classified the cellular solids in two groups, namely, stretching-dominated structures, which deform by uniaxial tension or compression of their struts, and bending-dominated structures (such as random foams), which deform by struts' bending. It was demonstrated that stretching-dominated structures possess superior mechanical properties. These analytical studies, while being informative, are limited to highly porous structures, where the struts can be assumed as slender beams. Apart from the numerical studies that consider lattices as a representative model for the foams, ${ }^{27)-30)}$ there are other numerical as well as experimental investigations focussing on mechanical behaviour and permeability of different lattices. ${ }^{12), 31)-35)}$ It is shown that a proper design of the morphology can result in structures with optimized properties for specific applications. ${ }^{26), 36), 37)}$ Such structures contain large numbers of geometrical details, which are impossible to generate with standard CAD packages. ${ }^{38)}$ In general, "classic" CAD tools are not able to perform quick and efficient design of lattice structures.

In this paper, we review several computational approaches, as well as new CAD tools, to design porous components with various unit-cells, using a library of purpose-built algorithms and scripts. These methods enable to generate structures with different features and functionalities, depending on the user's requirements. They were established during the last fifteen years spent in engineering porous components for different applications. Everything started from the attempt to generate realistic models of a stochastic foam for FEM studies, more complex approaches have been then adopted to improve performance and multi functionality. Structures' design was carried in parallel with AM. Indeed the combination of $\mathrm{CAD}$, simulation and $\mathrm{AM}$ introduced a new paradigm in complex ceramics' design. Finally several success stories are presented in the last paragraph.

\section{Stochastic foam design}

Foams are characterized by a random and non-periodic architecture. They exhibit scattered properties and determining their behaviour is difficult. One way is to measure
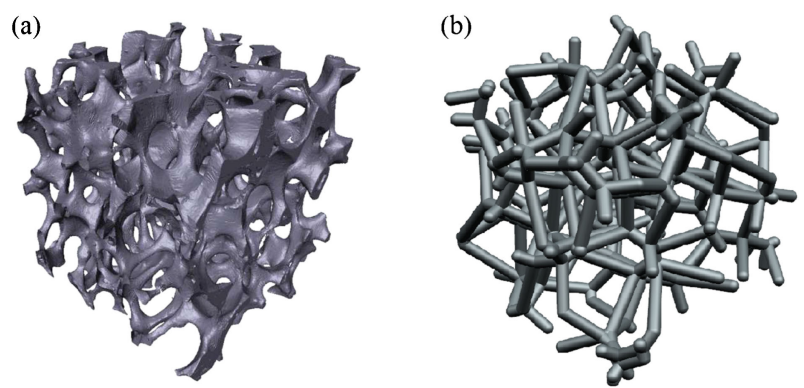

Fig. 1. (a) Ceramic foam reconstructed through the $\mathrm{XCT}^{25}{ }^{25}$ (b) The stochastic foam generated through the algorithm.

experimentally their effective properties, ${ }^{17)}$ else they can be calculated analytically ${ }^{2}$ or through finite element analysis. ${ }^{16)}$ The last method though, requires preparing a detailed CAD model of their geometry. A 3D numerical tool was developed in Matlab R2019a (MathWorks, Natick, Massachusetts, USA) for the generation of stochastic foams made up of struts elements. The script uses a list of nodes and edges obtained through a method which involves a X-ray Computed Tomography (XCT) scan of a real foam [Fig. 1(a)] and the skeletonization of the generated output file, (see Ref. 18 for further details). The data set contains also the connections (the edges) between the nodes. Setting the size of the sample as input, the algorithm randomly crops the skeletonized foam. The result is an array of nodes and edges ${ }^{39}$ ) that can be scaled in order to reach a certain dimension of the pores. The array is subsequently converted into a STEP file, which contains a set of spheres (centred in each node) and cylinders (with the edges as main axes). Spheres' and cylinders' diameter could be adjusted to define the foam porosity. Such STEP file can be easily imported in to a commercial CAD software for numerical simulations or foams' AM.

Foams are widely used in engineering applications, despite their diffusion, they show design limitations: they cannot be accommodated into any volume, they show local defects that reduce components' properties and above all, they are not reproducible. Figure 1 shows the difference between (a) a ceramic foam reconstructed through the $\mathrm{XCT}$ and (b) a stochastic foam generated through the algorithm.

\section{Structured lattice design}

There are unit-cells, with periodic boundaries, that can fill the space forming a lattice structure. The lattice can be made by polyhedra (e.g. cubic, dodecahedron, etc.) or by a combination of them (e.g. Weaire-Phelan ${ }^{40)}$ and octettruss). By replicating the unit-cell in three directions, a lattice structure is generated.

\subsection{Matlab approach}

A 3D numerical tool was developed in Matlab. It contains a library of several unit-cells, namely: Cube, Rotated Cube, Hexagonal, Octet-truss, Tetrakaidecahedron and Weaire-Phelan. The selected lattice is replicated in the space, forming datasets containing positions of the nodes 
Table 1. Type of cells for lattice structures

Rotated Cube Hexagonal

(a)
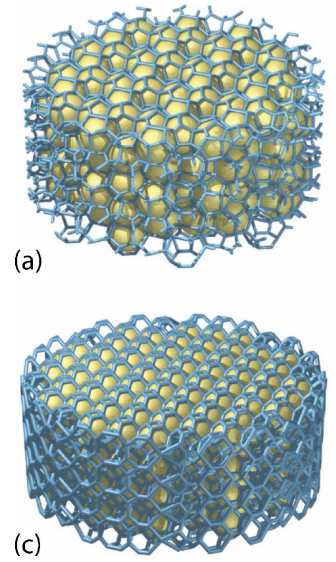
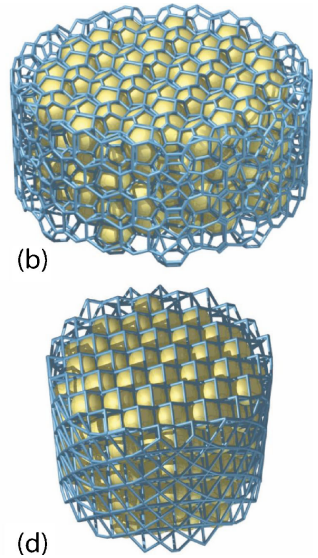

Fig. 2. Periodic structures made of: (a) Weaire-Phelan with open struts at the boundary, (b) Weaire-Phelan, (c) Tetrakaidecahedron and $(\mathrm{d})$ rotated cubic lattice with closed struts at the boundary. In the picture, the cells are filled with yellow spheres for better visibility of the structure.

and the connections. It is then possible to crop the lattice (usually in the form of a parallelepiped) into the desired shape. A drawback of this and the previous approaches is the presence of unconnected struts at the boundaries [Fig. 2(a)]. In many applications, this is a big issue while manufacturing, handling and operating the component. To avoid this, struts were connected with a second tool. Each set of points belonging to a clipped unit-cell on the boundaries (at the edge of the free struts) were identified and connected to each other by finding a convex shell containing those points. Figures 2(b)-2(d) depicts three lattice structures generated by this approach. The freeware Gnuplot $^{41)}$ and POV-Ray ray tracing tool ${ }^{42)}$ were used for their visualization.

The produced nodes and struts were then converted into a STEP file, made up of spheres (for each node) and cylinders (for each strut) with adjustable diameters. Such a STEP file can be easily imported in to a commercial CAD software for numerical simulations or AM.

\subsection{Grasshopper approach}

A parametric algorithm was developed into Rhinoceros (McNeel, Seattle, Washington, USA) using the Grasshopper plug-in. Grasshopper is a visual programming

language environment that allows creating programs by dragging components onto a canvas. The outputs to these components are then connected to the inputs of subsequent components. Grasshopper is primarily used to build generative algorithms, but its advanced uses include parametric modelling for structural engineering, ${ }^{43)}$ parametric modelling for architecture and fabrication, ${ }^{44)}$ lighting performance analysis for eco-friendly architecture and building energy consumption. ${ }^{45)}$ The algorithm contains a library of several unit-cells made up of lines (Table 1). In addition to the cells mentioned above, this code is able to manage other types of cells.

The desired lattice is selected and replicated in the space until filling the volume of the desired size and shape. The array of lines is then converted into a $3 \mathrm{D}$ triangular mesh, created using Cocoon add-on, ${ }^{46)}$ which uses a direct implementation of the Marching Cubes algorithm. ${ }^{47)}$ This component allows, through the triangulation of the space, to give a thickness to a line giving as input the distance from it (the radius of the strut). Therefore, the output is a STL file that can be processed immediately for 3D printing. The algorithm has several advantages with respect to the previous one: the generation time is much shorter, the software allows a real time visualization of the final structure and a real time properties calculation (surface area, volume, porosity, etc).

\section{Unstructured lattice design}

In many cases, an unstructured arrangement of the unit-cells is preferred in order to design lattices with heterogeneities such as variable cell size and orientation. Traditionally hexahedral and tetrahedral mesh elements have been used for discretization of numerical domains in computer simulations. ${ }^{48}$ ) Extracting the edges from an unstructured mesh can result in an equivalent unstructured lattice in a simple cubic or tetrahedral form. Extending this approach, one can extract the nodes and element connections from an unstructured hexahedral mesh and map a desired periodic unit cell into each mesh element. Any unit-cell with a cubic symmetry can be employed by this approach. Figure 3 shows some examples of the unit-cells with cubic symmetry.

Following this idea, a 3D Matlab tool was developed to receive a mesh file as the input and generate unstructured 

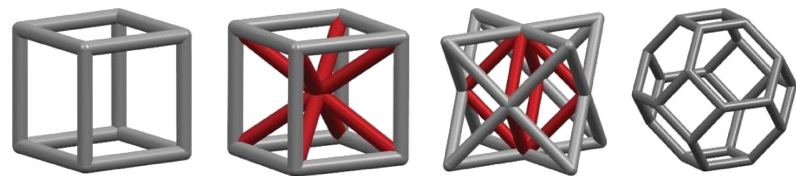

Fig. 3. Unit cells with cubic symmetry: cube, body centered cube, octet-truss and tetrakaidecahedron.
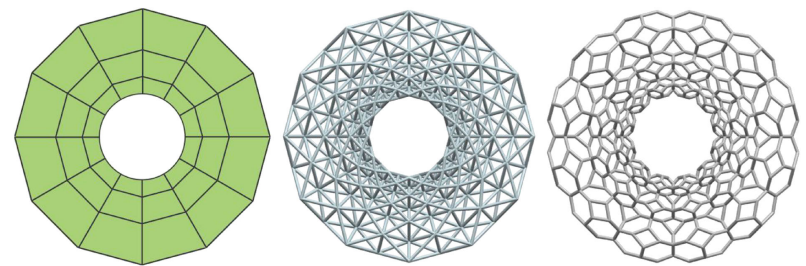

Fig. 4. From left to right: hexahedral mesh with a radial growth rate of 1.5 , used as the input for the code, generated CAD model with octet-truss unit-cells, and generated CAD model with tetrakaidecahedron unit-cells.

lattice structures with a certain unit cell as output. A hexahedral mesh of the part (which can be generated by a commercial software) can be used, onto which the scaled unit cell is mapped. Each strut diameter can be adjusted individually and different unit cells may be used, even for a single part. This allows generating unstructured lattices with variable unit cells and variable strut diameters.

Figure 4 demonstrates a simple example of a ring mesh with hexahedral elements, which is fed to the developed design tool as the input. Octet-truss and tetrakaidecahedron structures are synthesized and the results are converted into a STEP file containing spheres in place of each nodal point and cylinders presenting the struts of the lattice. The output STEP file can be then easily used for computer simulations or converted to an STL format for the $3 \mathrm{D}$ printing.

Figure 5 shows an example of lattices creation in complex forms with variable strut diameters. The generated cubic, tetrakaidecahedron and octet-truss lattice structures in Matlab are shown in Figs. 5(a)-5(c). These structures were then imported into Rhinoceros with the Grasshopper plug-in. The final geometries with variable strut diameters are made of triangular meshes, created using Cocoon add-on.

\section{Voronoi-based design}

Voronoi structures are obtained by partitioning space into zones based on the distance from a specific set of points. There are several algorithms to obtain Voronoi diagrams. ${ }^{49-51)}$ In order to create a Voronoi tessellation, first the desired enclosure is populated with a set of seed points. Based on these points, the space is then partitioned into small cells. Each cell contains the space around a seed point that is closer to that seed than any other one. Voronoi tessellations are widely used to describe the morphology of cellular structures. If they are created based on random seed points, they can represent the microstructural variability and irregularities in the open cell foams. Rhinoceros
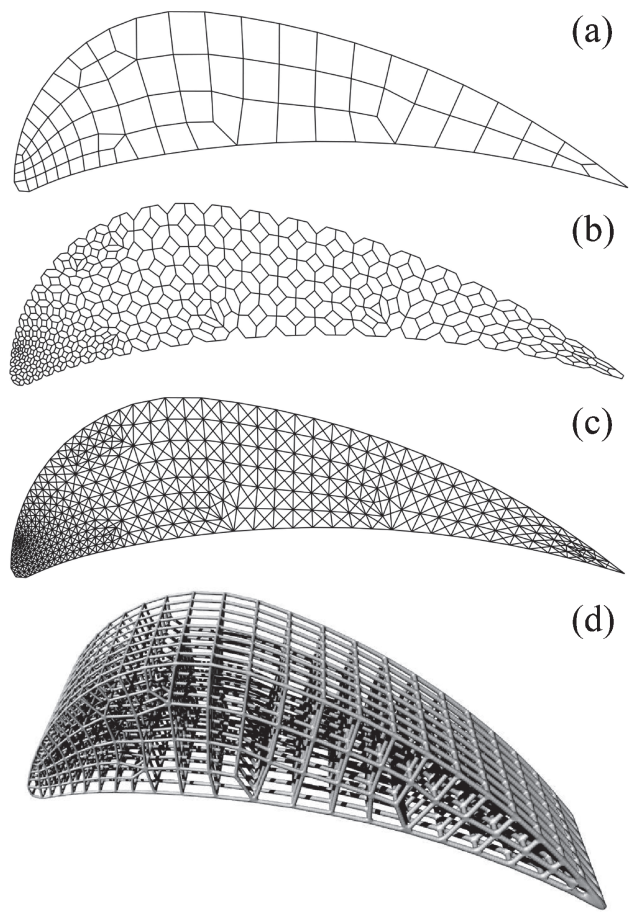

(d)

Fig. 5. 3D unstructured lattices in the form of an airfoil and variable cell sizes with (a) cubic, (b) tetrakaidecahedron and (c) octet-truss unit-cells. (d) Isometric view of the CAD model of the airfoil with cubic unit-cells and variable strut diameters.

with Grasshopper plug-in, was used for the implementation of Voronoi tessellations.

The code allows implementing a cells size gradient along one or more directions (Fig. 6). It is possible to generate a Voronoi structure with different porosities and pore size in the same volume.

The polyhedral structure generated by Voronoi depends on the locations of the seed points. Repetitive seed points' location will lead to repetitive lattice structures. We adopted what happens in nature at the grain boundaries of different crystals [in which spare atoms are randomly placed between the two (Fig. 7)] to generate a procedure to join different periodic architectures.

Periodic structures were generated using Voronoi algorithms. Figure 8(a) displays different lattices created with different input seeds. The flexibility of Voronoi tessellation can be employed to obtain regular lattices in complex shapes or even as a mean to "join" different structures. Figure 8(b) shows a TWO-DIMENSIONAL (2D) representation of a multi-lattice produced with this approach. The lattice contains a random Voronoi attached to quad, hexagonal and rotated quad lattices.

Extending this approach to 3D Voronoi diagrams, we obtained cubic, rotated cubic, hexagonal and tetrakaidecahedron lattice structures. Figure 9 shows a 3D multilattice consisting of a combination of rotated cube, cube, hexagon and random Voronoi cells, which are attached to a hexagonal honeycomb. Depending on the desired structure, the space is appropriately populated with seed points. The Voronoi algorithm then is used to create the structure at once. The created Voronoi structure is then decomposed 
(a)

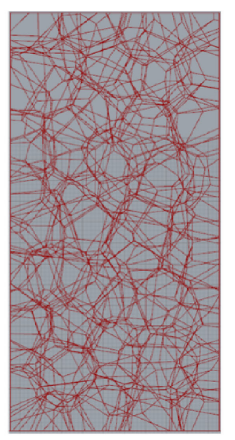

(b)

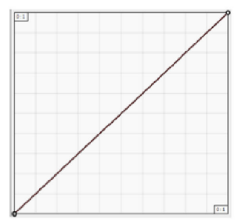

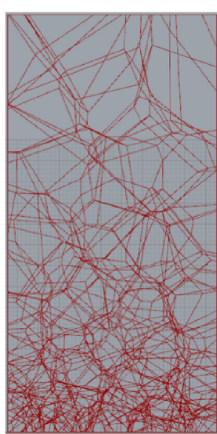

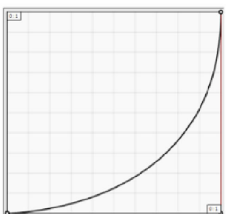

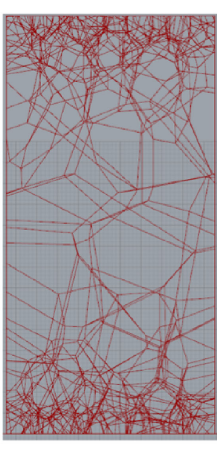
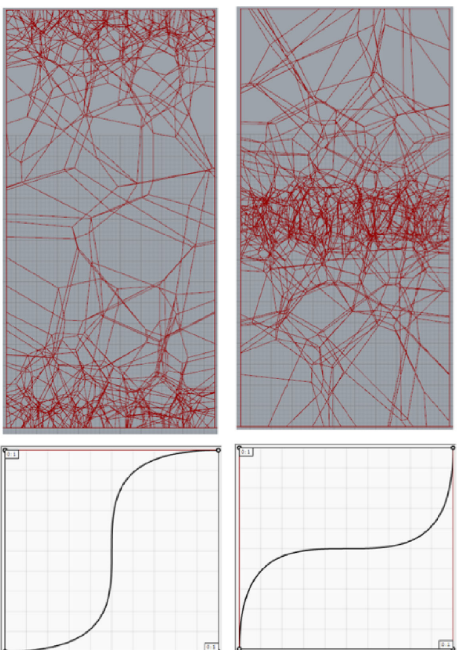

Fig. 6. Voronoi structure with variable cell size. (a) Voronoi cells edges. (b) Pore size distributions along $Y$ axis.

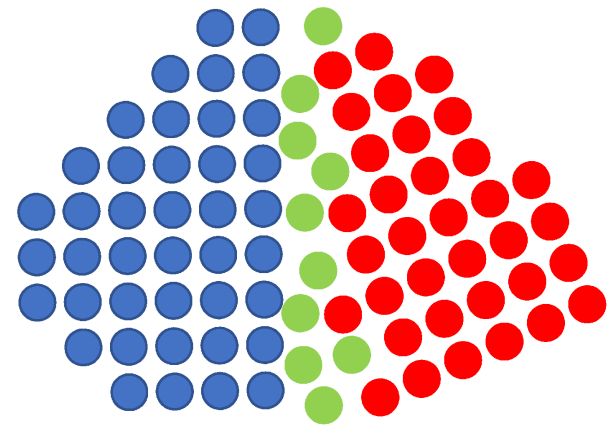

Fig. 7. Schematic of the random arrangement of atoms (green) at the grain boundary between two lattices of different orientation (red and blue).

(a)
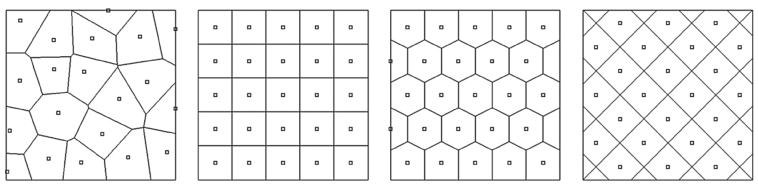

(b)

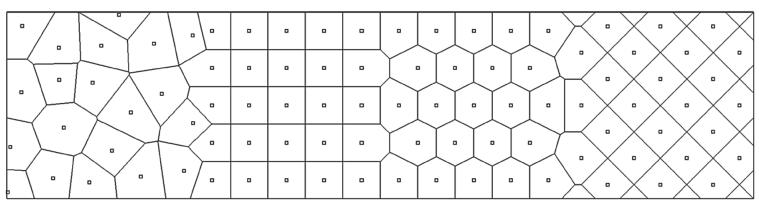

Fig. 8. (a) $2 \mathrm{D}$ representation of the generation of a Voronoi from different input seed points. From left to right: random, quad, hexagonal and rotated quad lattices. (b) 2D representation of a multi-lattice structure consisting of random Voronoi, quad, hexagonal and rotated quad cells.

to obtain the structure lines and surfaces (in case of a honeycomb). These nodes, edges and surfaces were then used to get CAD models.

\section{Applications}

In this paragraph we show how a CAD file, generated with the above-mentioned design approaches, is further turned into a real component for several engineering applications, each with a specific AM technique.
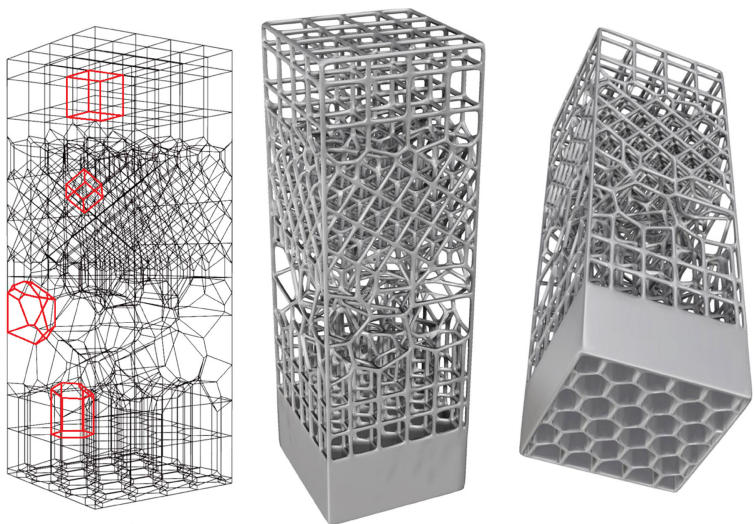

Fig. 9. Different views of a Voronoi-based structure as a combination of hexahedral, cubic, rotated cube and random cells (shown in red) attached to a hexahedral honeycomb.

\subsection{Porous burners}

Random foams produced by replica are commonly employed for the hot zone of porous burner. ${ }^{52)}$ The problem of standard foam was their reproducibility and uniformity due to the manufacturing method of the polymeric template. This was causing issues while handling, assembling and operating the burner. With the method in paragraph 3, it was possible to generate a structure which can solve the above mentioned issues. Figure 10 shows the porous burner in operation. The standard conditions for such a component are: working temperature $1350^{\circ} \mathrm{C}$, combustion environment air, $\mathrm{H}_{2} \mathrm{O}$ and volatile organic compounds. The EU project Energy Efficient Coil Coating Process (ECCO) aims at increasing the radiative power of such a component by increasing the operative temperature up to $1450{ }^{\circ} \mathrm{C}$ and optimizing the architecture's topology to improve radiation.

The method to convert the CAD file into and object was a hybrid methodology combining 3D printing of polymer replica with a slurry. ${ }^{10)}$ This process starts with rapid prototyping techniques, such as stereolithography (SLA) or selective laser sintering, for the production of three- 


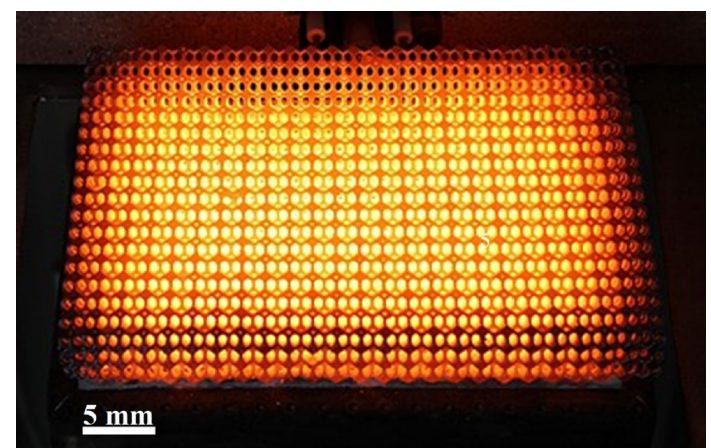

Fig. 10. Porous burner in operation.
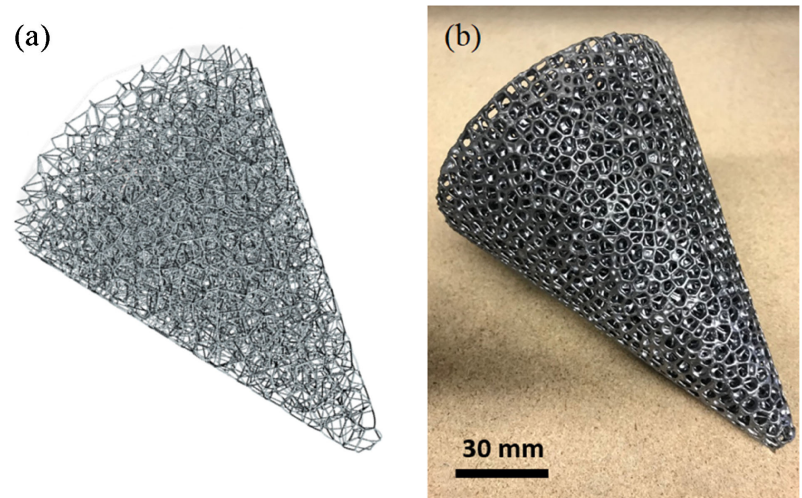

Fig. 11. Conical porous burner with Voronoi cells. (a) CAD model. (b) Fabricated structure.

dimensional polymeric parts. The printed body is dipped in a ceramic slurry to coat its surface till reaching a certain thickness. Then the body is placed thermally treated depending on the slurry's composition. This process allows the production of complex and high-performance components. The advantage of this technique is that the materials is added onto the existing polymeric surface and it does not possess the layered microstructure of $3 \mathrm{D}$ printed parts. This characteristic dramatically increases the mechanical strength of the porous architecture and, more importantly for this application, its thermal shock resistance.

Innovative type of porous burners were developed in the BioRoburPlus H 2020 research project ${ }^{53)}$ and in the $\mathrm{H} 2020$ research project ECCO project: ${ }^{54)} \mathrm{Si}-\mathrm{SiC}$ flat and conical prototypes, were fabricated by EngiCer SA (Viale Pereda 22, 6828 Balerna, Switzerland) with 10 PPI Voronoi open cells. This structure was designed using the algorithm in paragraph 5. The polymeric Voronoi cone was $3 \mathrm{D}$ printed, replicated with a SiC slurry and then infiltrated with molten silicon. Figure 11 shows the porous burner conical geometry. The conical burner showed a stable and complete combustion at low pollutant emissions from lowcaloric-value off-gases of the hydrogen production.

\subsection{Automotive catalyst substrates}

Automotive catalytic supports based on specifically designed engineered structures were developed, ${ }^{55)}$ in order to replace foams and standard honeycomb solutions, which cannot be engineered in terms of end user require-

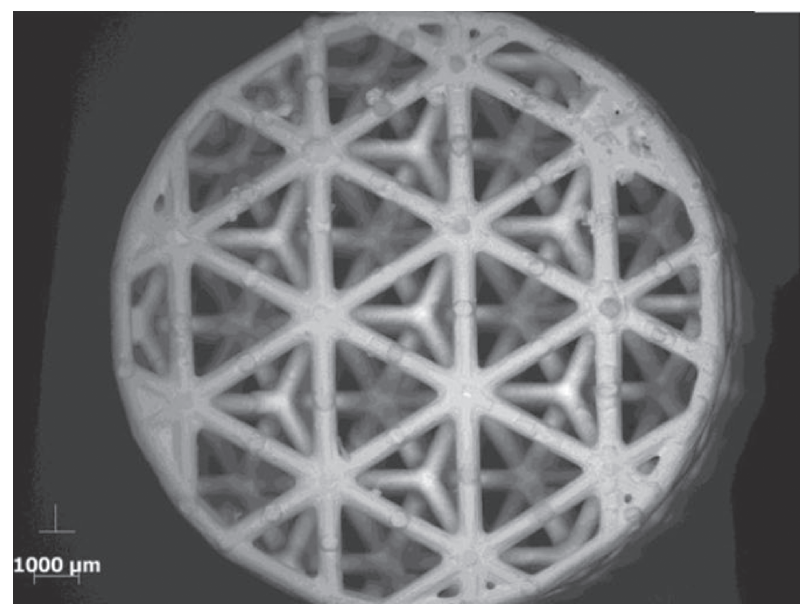

Fig. 12. Optical microscope image of the automotive catalytic support macroscopic features.

ments. Catalysts optimized in terms of their final requirements were generated using the approach in paragraph 3. Figure 12 shows the produced catalytic supports. Samples were produced in alumina and coated with $\gamma-\mathrm{Al}_{2} \mathrm{O}_{3}-\mathrm{Pt}$ suspension. Experiments have been conducted either at constant flow rates and increasing temperature profiles imposing a ramp from 50 to $600^{\circ} \mathrm{C}$ with a heating ramp of $10^{\circ} \mathrm{C} \mathrm{min}^{-1}$, in order to obtain the light-off curves, or at constant temperature but with increasing flow rate for understanding the impact of the flow velocity on reaction rates. ${ }^{56)}$

To produce these porous ceramics with a high surface area one needs to 3D print small cells with fine details. To our knowledge SLA is the technique with the highest resolution. SLA is an AM process used for the fabrication of three-dimensional complex parts through ultraviolet (UV) light to cure a polymer. This technique allows processing ceramic materials by cross linking a suspension of ceramic particles into a resin containing a photo initiator. ${ }^{57)}$ The object is built layer by layer thanks to the UV projections obtained by slicing the 3D-CAD file (.STL file) and the photopolymerization of the liquid system. Depending on the material used and the parts to be created, different printing parameters and setup can be used. ${ }^{58)}$ Once the part has been $3 \mathrm{D}$ printed, the polymerized resin is removed and sintered by thermal treatment. ${ }^{55)}$

\subsection{Heat exchangers}

Ceramic materials are widely used in applications that require high temperatures, such as heat exchangers. Through the use of engineered cellular architectures it is possible to enhance the heat transfer effectiveness and increase the performance of these systems. They can be inserted in tubular heat exchangers to exploits convection and radiation phenomena induced by the inner architecture. For this purpose, ceramic lattices with rotated cube cells of $4 \mathrm{~mm}$ were generated using the approach in paragraph 3 . Figure 13 shows the produced structures.

The lattices were fabricated with the SLA technique presented in the paragraph $6.2^{59)}$ and with the same ceram- 
(a)

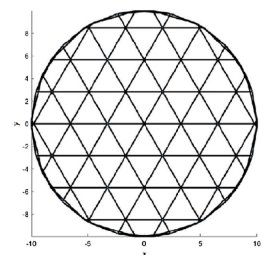

(b)

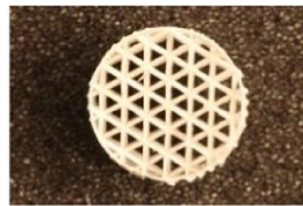

(c)

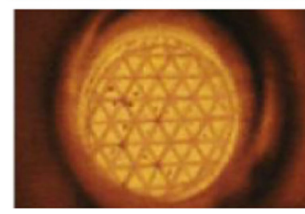

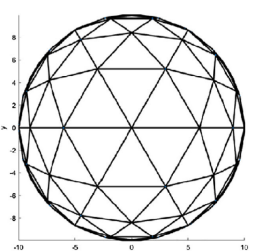
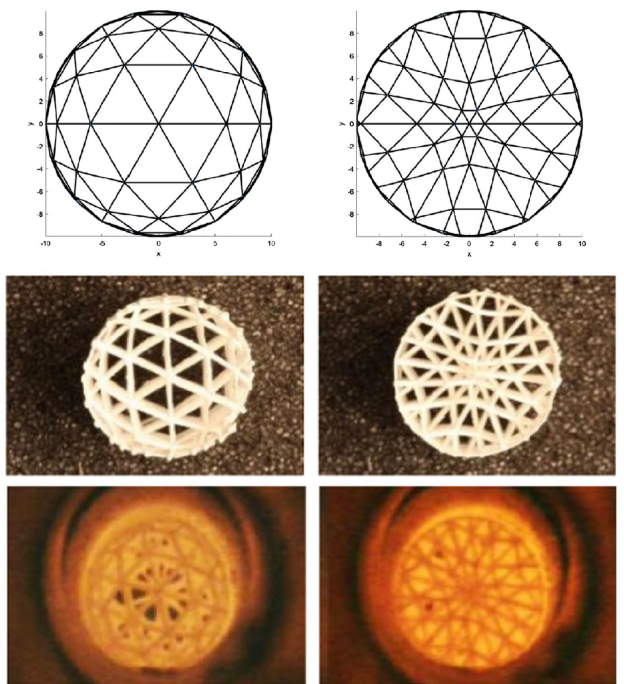

Fig. 13. Lattices to enhance the heat transfer in tubular heat exchangers. (a) CAD models made up of lines. (b) $3 \mathrm{D}$ printed geometries. (c) Components in operation.

ic slurry formulation. Different morphologies were employed in order to find the best solution for the heat transfer. The numerical and experimental studies shown that the morphologies of the lattice greatly influence the performance of the heat exchanger.

The heat transfer between flowing air and the tube was more effective if a lattice plug was used instead of an empty tube. Increases of performance (from 160 to $280 \%$ depending on the architecture) were achieved. Designs with large cells outside and small cells inside (Fig. 13 right), allows a higher fraction of thermal radiation to reach the central struts (better than with the other lattices) increasing therefore the heat transfer effectiveness.

\subsection{Solar receivers}

Tubular Si-infiltrated $\mathrm{SiC}_{\mathrm{f}} / \mathrm{SiC}$ composites were fabricated for solar receivers to enhance the performances of concentrated solar energy systems. ${ }^{60)}$ These ceramic materials, due to their unfavourable optical properties, cannot be $3 \mathrm{D}$ printed by SLA. Indirect AM technique such as binder jetting and FDM exist but, up to now, they do not have the same resolution of SLA. A hybrid methodology was proposed by combining 3D printing of polymer with slurry replica. ${ }^{10)}$ The designed structures per produced as per paragraph 6.1. In order to obtain the final ceramic product (Fig. 14) composed of an inner cellular ceramic and an outer dense ceramic matrix composite (CMC) skin, ${ }^{61)}$ the body was wrapped with filaments and then electrophoretic deposition (EPD) was performed.

It was not possible to test these components under operative conditions, but simulations ${ }^{61)}$ were performed. The results show that using 3D printed structures the tubes' performance can be increased significantly. Using a numerical model describing flow and heat transfer in a porous continuum the behaviour of a tubular receiver in closeto-service-environments has been predicted. The results show, that also in high temperature and high pressure environments the overall hat transfer can be increased by approximately 4 times if a porous in-lay is used.

\subsection{Catalytic supports for hydrogen produc- tion systems}

$\mathrm{Si}-\mathrm{SiC}$ periodic architectures based on rotated cube cells were produced for catalytic supports within the BioRobur European Horizon 2020 research project. ${ }^{62), 63)}$ The lattices were designed using the approach in paragraph 3 and 3D printed with a polymeric material. Replica with $\mathrm{SiC}$ slurry was performed and then the ceramic body was infiltrated with molten silicon. Figure 15 shows the catalytic supports.

In this project it was developed and tested a robust and efficient decentralized fuel processor based on the direct autothermal reforming (ATR) of biogas with a nominal production rate of $50 \mathrm{Nm}^{3} / \mathrm{h}$ of hydrogen and a plant efficiency of about $65 \%$. The ATR unit is composed of a structured catalyst support (Fig. 15) for the biogas reforming close coupled to a catalytic wall-flow filter to retain eventual soot particles.

The activation procedure was successful and a long duration test was carried out using a space velocity of $4000, \mathrm{~S} / \mathrm{C}=2.0, \mathrm{O} / \mathrm{C}=1.1$ and $\mathrm{T}_{\text {in }}=500^{\circ} \mathrm{C}$. A thermodynamic equilibrium and a methane conversion higher than $98 \%$ were achieved. The plant was able to reach the predicted conversions and concentrations at nominal capacities corresponding to $50 \mathrm{Nm}^{3} / \mathrm{h}(100 \mathrm{Kg} /$ day $)$ of pure hydrogen, creating a negligible pressure drop during the operation time of the processor.

\subsection{Thermal protection systems}

Replica method was used for the fabrication of innovative thermal management concepts for thermal protection of future space vehicles (THOR project). ${ }^{64), 65)}$ The lattices were designed using the algorithm in paragraph 4 with a particular and complex shape and then replicated with $\mathrm{SiC}$ slurry. Figure 16 shows the porous architecture of the core's thermal protection leading edge manufactured and 
(a)

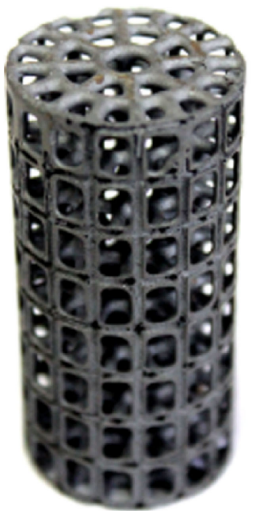

(b)

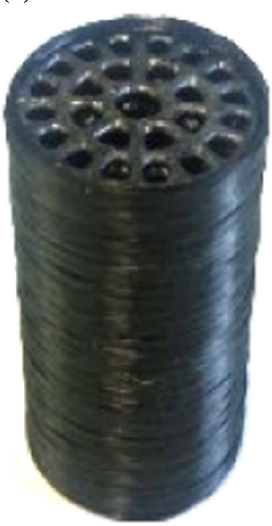

(c)

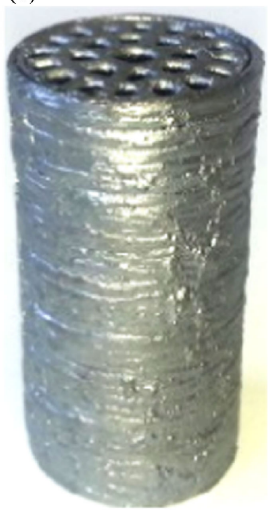

Fig. 14. Tubular Si-infiltrated $\mathrm{SiC}_{\mathrm{f}} / \mathrm{SiC}$ composites for solar receivers. (a) Periodic foam lattice by replica method combined with 3D printing. (b) After filament winding. (c) Final sample treated with EPD and Siinfiltration.

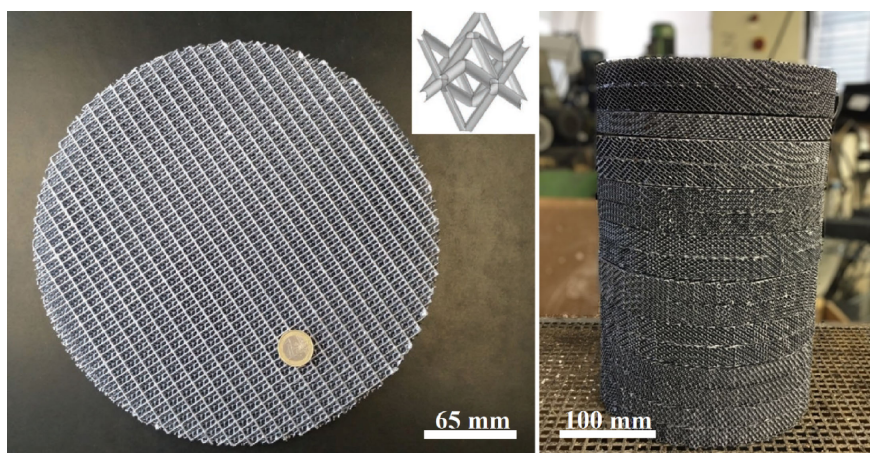

Fig. 15. Catalytic supports for hydrogen production systems.

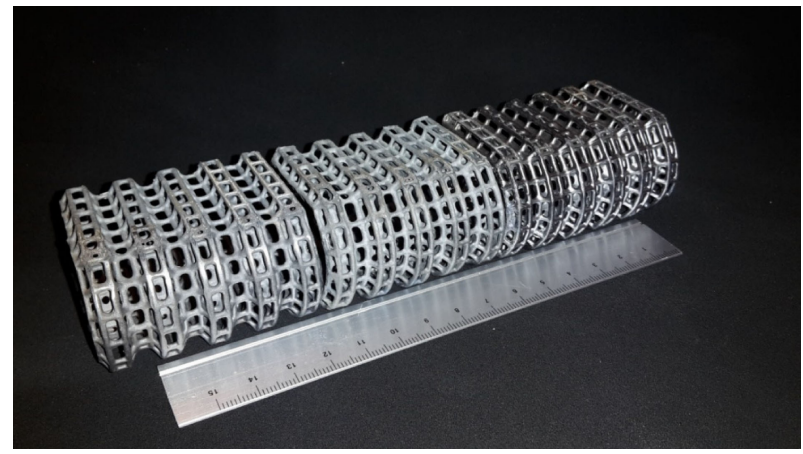

Fig. 16. Thermal protection system for future space vehicles: U-shaped porous ceramic architectures with hexagonal cells.

assembled at DLR (Köln, D).

An extensive test campaign was performed in a plasma wind tunnel at DLR. Operation and test conditions were selected to be representative for Earth re-entry with a reusable SiC-based TPS. With increasing coolant flow rate, temperatures decreases all along the surface. Largest differences were observed close to the stagnation point and at the downstream end of the lattice on the flat surface. Even at the lowest flow rate of $2.5 \mathrm{~g} / \mathrm{s}$ temperatures were below the results found for passively cooled reference configuration. The test results demonstrated the basic influence of active cooling on the thermal response of the leading edge to external heating.

\subsection{Micropollutant absorbers}

Ceramic architectures produced by AM and coated with natural zeolite were proposed as micropollutant adsorbers for the treatment of waste and drinking water. ${ }^{66)}$ The study demonstrated the potential of these structures used as a reusable filter. The three-dimensional parts were obtained using the algorithm in paragraph 3 and produced via SLA of a ceramic slurry. After sintering at $1600^{\circ} \mathrm{C}$, the $3 \mathrm{D}$ architectures were coated by replica, dipping each alumina support into a zeolite-based slurry for $15 \mathrm{~s}$ and centrifuged to remove the excess slurry. The coated supports were calcinated in a muffle furnace at $550{ }^{\circ} \mathrm{C}$ for $3 \mathrm{~h}$. Figure 17 shows the produced absorbers. For some filter a further surface modification was performed using surfactant treatments.

The adsorption of ten tested micro pollutants showed an overall efficiency of $41 \%$ on the as-coated filters and $43 \%$ on the further modified zeolite filters. Natural zeolite filters showed good specific adsorption capacity for metoprolol, citalopram, venlaflaxine and clarithromycin whereas the surface modified zeolite filters adsorb better hydrochlorothiazide, diclofenac, candesartan, mecoprop and irbesartan.

\section{Conclusions}

Due to the large number of new applications employing porous ceramics with targeted features and multiple functionalities, alternative design approaches are required to 


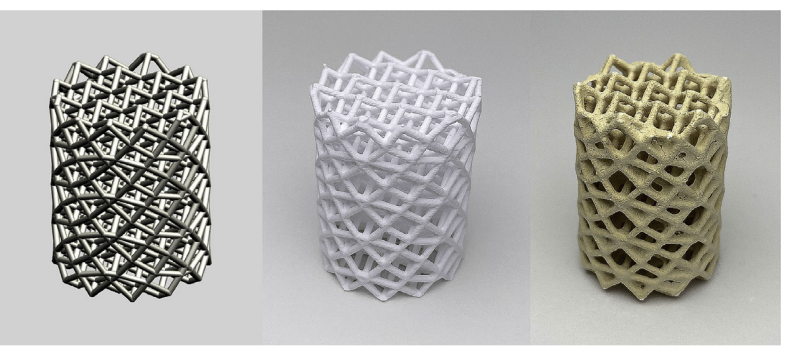

Fig. 17. Micropollutant absorbers. (a) CAD model. (b) 3D printed sample. (c) Coated support.

provide geometries for computer simulations and AM. This study reviews alternative design methods to create cellular structures with regular and irregular configurations. The developed tools were used to design components for several engineering applications: porous burners, heat radiators, catalyst carriers, solar absorbers, heat shields for space vehicles, water filters, automotive catalysts.

The structured lattice approach is based on the creation of a desired unit-cell and its spatial replication within a geometrical enclosure followed by cutting the struts that "fall out" of the enclosure boundaries. This approach, while being simple, provided the tool used for the creation of most of the components shown in this work. It is an ideal tool for generation of regular lattices in simple forms such as a cylinder for heat exchangers or gas filters. However, it lacks the flexibility to create structures with variable cell sizes, variable unit-cells, or complex shapes.

The unstructured lattice approach was developed in order to create lattices with complex enclosures. The idea is based on mapping lattices with cubic symmetry within hexahedral mesh elements. The developed design tool imports a part meshed by commercial software, and creates unit-cells within each hexahedral mesh element. The developed numerical tool can generate lattices with variable cell size within any complex shape. However, it only can use unit-cells with cubic symmetry. Furthermore, the mesh generation must be carefully performed in order to avoid elements with large distortions.

The Voronoi approach is based on the creation of multifunctional structures with different input seed points. Here we show for the first time that using patterned seed points for a Voronoi algorithm, cubic, tetrakaidecahedral and hexagonal lattices can be generated in any desired direction. This tool provides a flexible way to design pieces consisting of different lattice types, in different orientations, and with variable cell sizes. It can be used to join two lattices, through a Voronoi-based mid-layer between them. However, the approach is limited to certain unit-cells. A further development of the tool is possible in order to combine this tool with the second approach to include further unit-cells with cubic symmetry.

Acknowledgments The development of the applications shown in this paper has received funding from several EU and Swiss national projects over the last years.

The porous burners applications shown here were developed within two projects which have received funding from the European Union's Horizon 2020 research and innovation under grant agreement No 736272 (BioRoburPlus) and grant agreement 768692 (ECCO).

\section{References}

1) G. Mayer and M. Sarikaya, Exp. Mech., 42, 395-403 (2002).

2) L. J. Gibson and M. F. Ashby, "Cellular solids: structure and properties", Cambridge university press (1999).

3) T. Fend, Opt. Appl., 40, 271-284 (2010).

4) S. Gianella, D. Gaia and A. Ortona, Adv. Eng. Mater., 14, 1074-1081 (2012).

5) Y. S. Montenegro Camacho, S. Bensaid, S. Lorentzou, N. Vlachos, G. Pantoleontos, A. Konstandopoulos, M. Luneau, F. C. Meunier, N. Guilhaume, Y. Schuurman, E. Werzner, A. Herrmann, F. Rau, H. Krause, E. Rezaei, A. Ortona, S. Gianella, A. Khinsky, M. Antonini, L. Marchisio, F. Vilardo, D. Trimis and D. Fino, E. Werzner, Int. J., 43, 161-177 (2018).

6) A. Szczurek, A. Ortona, L. Ferrari, E. Rezaei, G. Medjahdi, V. Fierro, D. Bychanok, P. Kuzhir and A. Celzarda, Carbon, 88, 70-85 (2015).

7) E. Rezaei, S. Haussener, S. Gianella and A. Ortona, Ceram. Int., 42, 16255-16261 (2016).

8) A. R. Studart, U. T. Gonzenbach, E. Tervoort and L. J. Gauckler, J. Am. Ceram. Soc., 89, 1771-1789 (2006).

9) Y. Conde, J. F. Despois, R. Goodall, A. Marmottant, L. Salvo, C. San Marchi and A. Mortensen, Adv. Eng. Mater., 8, 795-803 (2006).

10) A. Ortona, C. D'Angelo, S. Gianella and D. Gaia, Mater. Lett., 80, 95-98 (2012).

11) J. W. Halloran, Annu. Rev. Mater. Res., 46, 19-40 (2016).

12) J. T. Muth, P. G. Dixon, L. Woish, L. J. Gibson and J. A. Lewis, P. Natl. Acad. Sci. USA, 114, 1832-1837 (2017).

13) L. Schlier, W. Zhang, N. Travitzky, P. Greil, J. Cypris and M. Weclas, Int. J. Appl. Ceram. Tec., 8, 1237-12 (2011).

14) S. Bose, S. Vahabzadeh and A. Bandyopadhyay, Mater. Today, 16, 496-504 (2013).

15) M. Mott and J. R. Evans, J. Am. Ceram. Soc., 84, 307313 (2001).

16) S. Pusterla, A. Ortona, C. D'Angelo and M. Barbato, J. Porous Mat., 19, 307-315 (2012).

17) C. D'Angelo, A. Ortona and P. Colombo, Acta. Mater., 60, 6692-6702 (2012).

18) C. D'Angelo, A. Ortona and P. Colombo, Acta. Mater., 61, 5525-5534 (2013).

19) M. Pelanconi and A. Ortona, Materials, 12.24, 4134 (2019).

20) O. Al-Ketan, M. Pelanconi, A. Ortona and R. K. Abu Al-Rub, J. Am. Ceram. Soc., 102, 6176-6193 (2019).

21) A. G. Evans, J. W. Hutchinson and M. F. Ashby, Curr. Opin. Solid St. M., 3, 288-303 (1998).

22) L. Giani, G. Groppi and E. Tronconi, Ind. Eng. Chem. Res., 44, 4993-5002 (2005).

23) M. Lacroix, P. Nguyen, D. Schweich, C. P. Huu, S. Savin-Poncet and D. Edouard, Chem. Eng. Sci., 62, 3259-3267 (2007).

24) J. T. Richardson, Y. Peng and D. Remue, Appl. Catal. AGen., 204, 19-32 (2000).

25) A. Ortona and E. Rezaei, Adv. Sci. Tech., 91, 70-78 
(2014).

26) V. S. Deshpande, M. F. Ashby and N. A. Fleck, Acta. Mater., 49, 1035-1040 (2001).

27) S. Cunsolo, M. Iasiello, M. Oliviero, N. Bianco, W. K. Chiu and V. Naso, J. Heat Transf., 138, 022601-022608 (2016).

28) W. Xu, H. Zhang, Z. Yang and J. Zhang, J. Porous Mat., 16, 65-71 (2009).

29) P. Thiyagasundaram, J. Wang, B. V. Sankar and N. K. Arakere, Eng. Fract. Mech., 78, 1277-1288 (2011).

30) W. Y. Jang, S. Kyriakides and A. M. Kraynik, Int. J. Solids Struct., 47, 2872-2883 (2010).

31) S. Truscello, G. Kerckhofs, S. Van Bael, G. Pyka, J. Schrooten and H. Van Oosterwyck, Acta Biomater., 8, 1648-1658 (2012).

32) Z. C. Eckel, C. Zhou, J. H. Martin, A. J. Jacobsen, W. B. Carter and T. A. Schaedler, Science, 351, 58-62 (2016).

33) D. Ali and S. Sen, J. Mech. Behav. Biomed. Mater., 75, 262-270 (2017).

34) P. F. Egan, V. C. Gonella, M. Engensperger, S. J. Ferguson and K. Shea, PLoS One, 12, e0182902 (2017).

35) H. Cui, R. Hensleigh, H. Chen and X. Zheng, J. Mater. Res., 33, 360-371 (2018).

36) L. E. Murr, S. M. Gaytan, F. Medina, H. Lopez, E. Martinez, B. I. Machado, D. H. Hernandez, L. Martinez, M. I. Lopez, R. B. Wicker and J. Bracke, Philos. Trans. R. Soc., A, 368, 1999-2032 (2010).

37) P. Wang, J. B. Li, K. Vafai, L. Zhao and L. Zhou, J. Sol. Energy Eng., 139, 051012-051017 (2017).

38) H. Wang, Y. Chen and D. W. Rosen, "A hybrid geometric modeling method for large scale conformal cellular structures", In ASME 2005 International Design Engineering Technical Conferences and Computers and Information in Engineering Confer, September (2005).

39) G. Bianchi, S. Gianella and A. Ortona, J. Ceram. Sci. Technol., 8, 59-66 (2017).

40) R. Kusner and J. M. Sullivan, Forma, 11, 233-242 (1996).

41) T. Williams and C. Kelley, "GNUplot: an interactive plotting program", http://www.gnuplot.info (1998).

42) "POV-Ray", Persistence of Vision Pty. Ltd., Williamstown, Victoria, Australia, http://www.povray. org/.

43) O. Georgiou, P. Richens and P. Shepherd, "Performance based interactive analysis", In Computational Design Modelling, Springer, Berlin, Heidelberg (2011) pp. 115-122.

44) A. Tedeschi, "Parametric architecture with Grasshopper®: primer”, Le Penseur (2011).

45) D. Willis, W. W. Braham, K. Muramoto and D. A. Barber, "Energy accounts: Architectural representations of energy, climate, and the future", Routledge (2016).

46) Stasiuk., Cocoon. ADDON. Version 1. Released on 2015-Jul-22. Provides 6 components. Created by David.

47) P. Bourke, Polygonising a scalar field Also known as: "3D Contouring", "Marching Cubes", "Surface Reconstruction”, http://local.wasp.uwa.edu.au/ pbourke/ geometry/polygonise (1994).

48) D. S. Lo, "Finite element mesh generation", CRC Press (2014).

49) S. Fortune, Algorithmica, 2.1-4, 153 (1987).
50) J. Martínez, J. Dumas and S. Lefebvre, ACM Trans. Graph., 35, 1-12 (2016).

51) D. M. Yan, W. Wang, B. LéVy and Y. Liu, Comput. Aided Des., 45, 843-852 (2013).

52) A. Ortona, S. Pusterla, P. Fino, F. R. A. Mach, A. Delgado and S. Biamino, Adv. Appl. Ceram., 109, 246251 (2010).

53) BioRoburPlus European Horizon 2020 research project, "Advanced direct biogas fuel processor for robust and cost-effective decentralised hydrogen production", https://www.bioroburplus.org/.

54) Energy Efficient Coil Coating Process https://www. spire2030.eu/ecco. [Online] SPIRE (2017).

55) O. Santoliquido, G. Bianchi, P. Dimopoulos Eggenschwiler and A. Ortona, Int. J. Appl. Ceram. Tec., 14, 1164-1173 (2017).

56) V. Papetti, P. D. Eggenschwiler, A. Della Torre, F. Lucci, A. Ortona and G. Montenegro, Int. J. Heat Mass Tran., 126, 1035-1047 (2018).

57) O. Santoliquido, P. Colombo and A. Ortona, J. Eur. Ceram. Soc., 39, 2140-2148 (2019).

58) O. Santoliquido, G. Bianchi and A. Ortona, "Additive Manufacturing of Complex Ceramic Architectures", In International Conference on Additive Manufacturing in Products and Applications, September, Springer, Cham. (2017) pp. 117-123.

59) M. Pelanconi, M. Barbato, S. Zavattoni, G. L. Vignoles and A. Ortona, Mater. Des., 163, 107539 (2019).

60) A. Ortona, T. Fend, H. W. Yu, K. Raju, P. Fitriani and D. H. Yoon, Sol. Energy Mater. Sol. Cells, 132, 123130 (2015).

61) A. Ortona, D. H. Yoon, T. Fend, G. Feckler and O. Smirnova, Sol. Energy Mater. Sol. Cells, 140, 382-387 (2015).

62) A. Herrmann, M. Pönisch, E. Werzner, M. Laurinat, E. Rezaei, A. Ortona, S. Bensaid, D. Fino and D. Trimis, "Development of an autothermal biogas processor for hydrogen production", In Proc. of the International Gas Union Research Conference (IGRC 201, September (2014).

63) Y. S. Montenegro Camacho, S. Bensaid, D. Fino, D. Trimis, A. Herrmann, N. Guilhaume, Y. Schuurman, A. G. Konsatndopoulos, S. Lorentzou, S. Gianella, M. Antonini, L. Marchisio, A. Ortona and A. Khinsky, WIT Trans. Ecol. Environ., 195, 463-474 (2015).

64) B. Esser, J. Barcena, M. Kuhn, A. Okan, L. Haynes, S. Gianella, A. Ortona, V. Liedtke, D. Francesconi and H. Tanno, J. Spacecraft Rockets, 53, 1051-1060 (2016).

$65)$ L. Ferrari, M. Barbato, B. Esser, I. Petkov, M. Kuhn, S. Gianella, J. Barcena, C. Jimenez, D. Francesconi, V. Liedtke and A. Ortona, "Sandwich structured ceramic matrix composites with periodic cellular ceramic cores: An active cooled thermal protection for space vehicles", Composite Structu (2016).

66) R. König, M. Spaggiari, O. Santoliquido, P. Principi, G. Bianchi and A. Ortona, J. Clean. Prod., 258, 120500 (2020).

67) M. Spaggiari, G. Bianchi, O. Santoliquido, P. Senn, P. Fitriani, Y. De Hazan, D. Penner, D.-H. Yoon and A. Ortona, Ceram. Int., 45, 15603-15608 (2019). 